\title{
Identifying the Molecular Origins of High- Performance in Organic Photodetectors Based on Highly Intermixed Bulk Heterojunction Blends
}

Saurav Limbu, ${ }^{\dagger, \#}$ Kyung-Bae Park, ,\# Jiaying Wu, $\$$ Hyojung Cha, ${ }^{\S}$ Sungyoung Yun, , SeonJeong Lim,,+ Hao Yan, Joel Luke, Gihan Ryu, Chul-Joon Heo, ${ }^{\dagger}$ Sunghan Kim, Yong Wan

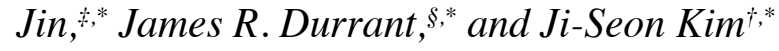

Department of Physics and Centre for Processable Electronics, Imperial College London, London, SW7 2AZ, UK. :Organic Materials Lab, Samsung Advanced Institute of Technology, Samsung Electronics Co. Ltd., Samsung-ro, Yeongtong-gu, Suwon-si, Gyeonggi-do, 16678, Korea. ${ }^{\S}$ Department of Chemistry and Centre for Processable Electronics, Imperial College London, London, SW7 2AZ, UK. "Equal contribution. 


\section{SUPPORTING EXPERIMENTAL SECTION}

Synthesis of the novel p-type naphthalene based small molecule IDDSe. The synthesis of IDDSe (chemical name: 5- (naphthalen-1-yl (phenyl) amino) selenophen-2-yl) methylene) 1H-indene-1,3 (2 H) -dione) follows the following procedures: To a mixture of 5-(naphthalen1-yl(phenyl)amino)selenophene-2-carbaldehyde $(1 \mathrm{mmol})$ and $1 \mathrm{H}$-indene-1,3(2H)-dione $(1.1$ $\mathrm{mmol})$; an anhydrous ethanol $(\mathrm{EtOH})(20 \mathrm{~mL})$ was added at room temperature. The reaction mixture was stirred at the room temperature for $30 \mathrm{~min}$, to which a drop of piperidine was added by using a syringe. The reaction mixture was further stirred at $85^{\circ} \mathrm{C}$ for $6 \mathrm{hrs}$ and cooled to room temperature. After the mixture was quenched with $\mathrm{H}_{2} \mathrm{O}$, the resulting solid was collected via filtration and thoroughly washed with $\mathrm{H}_{2} \mathrm{O}$ and hexanes, to obtain reddish orange solid. The solid products were further purified via column chromatography on silica gel (chloroform/ethyl acetate $=9 / 1$ ). The resulting product was recrystallized from dichloromethane/hexanes (yield $=93 \%$ ).

Melting point: $276^{\circ} \mathrm{C} .1 \mathrm{H}-\mathrm{NMR}(600 \mathrm{MHz}$, Methylene Chloride-d2): $\delta$ 8.08-7.99 (m, 3H), $7.84(\mathrm{~s}, 1 \mathrm{H}), 7.81-7.74(\mathrm{~m}, 2 \mathrm{H}), 7.70(\mathrm{dd}, \mathrm{J}=6.0,2.1 \mathrm{~Hz}, 1 \mathrm{H}), 7.69-7.54(\mathrm{~m}, 8 \mathrm{H}), 7.48-$ $7.40(\mathrm{~m}, 2 \mathrm{H}), 7.32$ (t, J = 7.5 Hz, 1H), 6.39 (d, J = 5.0 Hz, 1H). 13C-NMR (600 MHz, Methylene Chloride-d2): $\delta 191.20,191.14,178.05,151.29,146.56,142.41,141.84,140.79$, $140.21,135.96,134.36,134.29$, 130.54, 130.25, 130.07, 129.43, 128.20, 128.00, 127.48, 127.47, 126.85, 124.79, 123.49, 122.28, 122.13, 117.17, 114.37. HRMS (ESI+): calculated for $\mathrm{C} 30 \mathrm{H} 20 \mathrm{NO} 2 \mathrm{Se}[\mathrm{M}+\mathrm{H}+]$ : 506.0659; found: 506.0652. Elemental analysis: calculated for C30H19NO2Se: $\mathrm{C}-71.43 \%, \mathrm{H}-3.80 \%, \mathrm{~N}-2.78 \%$; found: $\mathrm{C}-71.55 \%, \mathrm{H}-3.72 \%, \mathrm{~N}-$ $2.86 \%$. 


\section{Note S1: Kinetic model for calculating CT state binding energy $\left(E_{a}\right)$ and size $(d)$.}

Firstly, the photoluminescence quenching efficiency $(P L q E)$ as a function of temperature is given by: $P L q E(T)=\frac{P L_{\text {initial } T}-P L_{T}}{P L_{\text {initial }}}$; where $P L_{\text {initial } T}$ represents $P L$ at initial temperature and $P L_{T}$ represents PL at desired temperatures. Similar equation is used for $P L q E(V)$ i.e. as a function of bias voltage.

\section{(A) Calculation of CT state binding energy $\left(E_{a}\right)$}
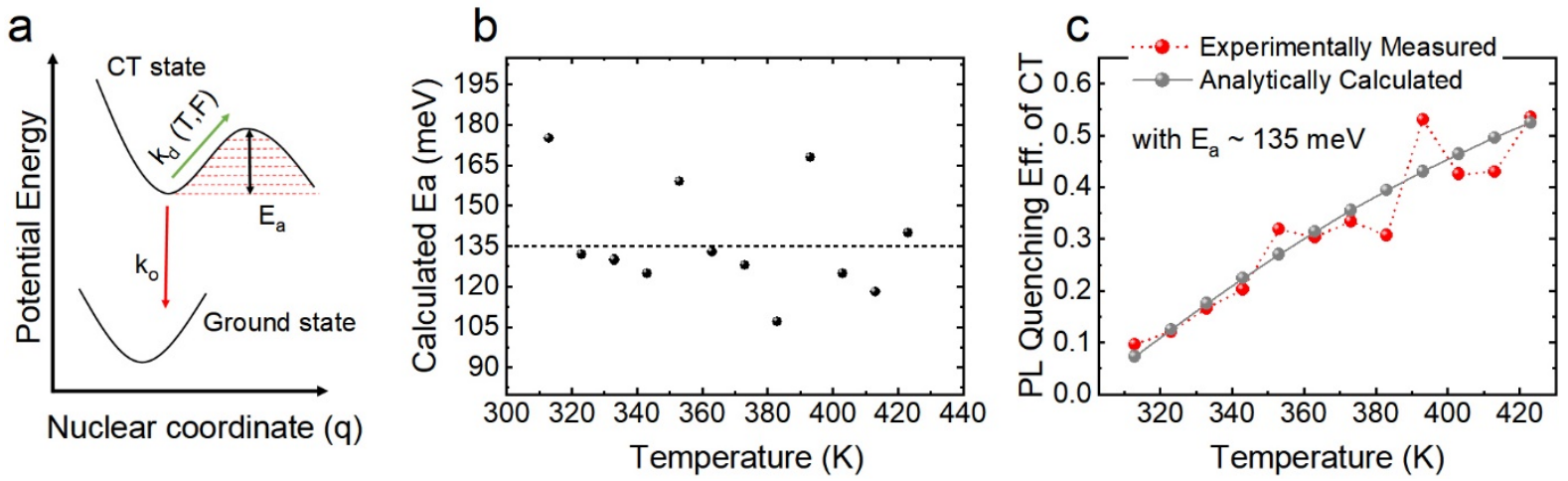

Figure S1 (a) Copy of Figure 5d, illustration for kinetic model of CT state dissociation. (b) Analytically calculated $E_{a}$ across a range of temperatures using Equation 5. The dotted line shows average value: $136 \pm 6 \mathrm{meV}$ (c) Analytically calculated PLqE via Equation 6, with $E_{a}=135 \mathrm{meV}$. It shows a close agreement with experimentally measured PLqE (shown in Figure 5f) as a function of temperature.

The CT dissociation efficiency at $0 \mathrm{~V}$ in room temperature (RT) is given by (refer Figure S1a):

$$
\frac{k_{d}}{k_{0}+k_{d 0}}=0.3(E Q E \text { at } 0 \mathrm{~V}, R T)
$$

where, $k_{0}$ is the CT state to ground state recombination rate and $k_{d}$ is the dissociation rate. The $\mathrm{CT}$ dissociation efficiency of 0.3 is taken from EQE at $0 \mathrm{~V}$ in room temperature (Figure $4 \mathrm{~b}$ ). Assumptions: bimolecular recombination is negligible and EQE is determined by $\mathrm{CT}$ dissociation efficiency. Thus, we have:

$\left[k_{d 0}=k_{d}\right.$ at $0 \mathrm{~V}$ and $\left.\mathrm{RT}\right] ;\left[k_{0}+k_{d 0}=1 / 800 \mathrm{ps}=1.25 n \mathrm{~s}^{-1}\right]$; where, $k_{0}+k_{d 0}$ is $\mathrm{CT}$ state recombination rate obtained from transient absorption kinetics (Figure 1e). So, the Equation 1 can be written as: $\left[3.34 k_{d 0}=k_{0}+k_{d 0}=1.25 n s^{-1}\right] ;\left[\therefore k_{d 0}=\right.$ $\left.0.37 n s^{-1}\right] ;\left[k_{0}=0.88 n s^{-1}\right]$ 
At $150^{\circ} \mathrm{C}$, the total $\mathrm{CT}$ decay pathway is as follows:

$$
\left(1-E Q E_{0 V}\right) \times\left(1-P L Q E_{T=150{ }^{\circ} \mathrm{C}}\right)=(1-0.3) \times(1-0.54)=0.7 \times 0.46=0.32
$$

Here, the $P L Q E_{T=150{ }^{\circ} \mathrm{C}}$ is obtained experimentally from Figure 5f. So, the CT dissociation yield is: $1-0.32=0.68$. Thus, at $150{ }^{\circ} \mathrm{C}$, the $\mathrm{CT}$ dissociation rate is:

$$
\frac{k_{d}}{k_{0}+k_{d}}=0.68\left(\text { at } 0 \mathrm{~V}, 150^{\circ} \mathrm{C}\right)
$$

So, $\left[1.47 k_{d}=k_{0}+k_{d}\right] ;\left[1.47 k_{d}=0.88+k_{d}\right] ;\left[\therefore k_{d}=1.87 n s^{-1}\right]$

Now, the CT binding energy $\left(E_{a}\right)$ of the rate constant $k_{d}$ is given by Arrhenius equation:

$$
k_{d}=A \exp \left(\frac{-E_{a}}{k_{B} T}\right)
$$

where, $k_{B}$ is the Boltzmann's constant, $T$ is the temperature and $A$ is the pre-exponential frequency factor. $E_{a}$ can be calculated by ratio of dissociation rate constant at room temperature and $150{ }^{\circ} \mathrm{C}$ (i.e. $k_{d 0}=0.37 n s^{-1}$ from Equation 1 and $k_{d}=1.87 n s^{-1}$ from Equation 2 ) as follows:

$$
\left[\frac{k_{d}}{k_{d 0}}=\frac{A \exp \left(\frac{-E_{a}}{k_{B} \cdot 423}\right)}{A \exp \left(\frac{-E_{a}}{k_{B} \cdot 298}\right)}\right] ;\left[\ln \frac{k_{d}}{k_{d 0}}=\ln \frac{1.87}{0.37}=-\frac{E_{a}}{k_{B} \cdot 423}+\frac{E_{a}}{k_{B} \cdot 298}=1.62\right] ;\left[\therefore E_{a} \sim 140 \mathrm{meV}\right]
$$

Likewise, average CT state binding energy $\left(E_{a}\right)$ across various temperatures can be calculated to obtain statistically relevant value for $E_{a}$.

$P L q E(T)$ referenced to room temperature is given by:

$$
\text { Dissociation yield }=0.3+0.7 \cdot \operatorname{PLQE}(T)=\frac{k_{d}}{k_{0}+k_{d}}
$$

Thus, the dissociation rate $k_{d}$, referenced to room temperature using the Arrhenius equation (Equation 3) is given as: $\frac{k_{d}}{k_{d 0}}=\exp \left\{\frac{-E_{a}}{k_{B}}\left(\frac{1}{T}-\frac{1}{298}\right)\right\}$

Here putting $k_{0} \approx 2.4 k_{d 0}$ we get: $k_{0}=\frac{2.4 k_{d}}{\exp \left\{\frac{-E_{a}}{k_{B}}\left(\frac{1}{T}-\frac{1}{298}\right)\right\}}$

Putting expression of $k_{0}$ in Equation $4: \frac{k_{0}+k_{d}}{k_{d}}=1+\frac{2.4 k_{d}}{k_{d} \cdot \exp \left\{\frac{-E_{a}}{k_{B}}\left(\frac{1}{T}-\frac{1}{298}\right)\right\}}=\frac{1}{0.3+0.7 \cdot \operatorname{PLqE}(T)}$

Solving the above equation, we get analytical expression for $E_{a}$ as follows:

$$
E_{a}=k_{B} \cdot \frac{\ln \left\{\left(\frac{1}{0.3+0.7 \cdot P \operatorname{LqE}(T)}-1\right) \cdot 0.42\right\}}{\left(\frac{1}{T}-\frac{1}{298}\right)}
$$


Figure $\mathrm{S} 1 \mathrm{~b}$ shows the analytically calculated $E_{a}$ across the range of temperatures, with average value of $136 \pm 6 \mathrm{meV}$, i.e. $\sim 135 \mathrm{meV} \sim 5 \mathrm{kBT}$ (at $\mathrm{T}=298 \mathrm{~K}$ ). Likewise, the PLqE(T) can be back calculated from Equation 4 and 5 as follows (also refer to Figure S1c):

$$
P L Q E(T)=\left(\frac{0.42}{\exp \left\{\frac{E_{a}}{k_{B}}\left(\frac{1}{T}-\frac{1}{298}\right)\right\}+0.42}-0.3\right) \cdot \frac{1}{0.7}
$$

\section{(B) Calculation of CT state size (d)}

At $-5 \mathrm{~V}:\left(1-E Q E_{0 V}\right)\left(1-P L Q E_{-5 V}\right)=(1-0.3)(1-0.58)=0.29$

The $E Q E_{0 V}$ and $P L Q E_{-5 V}$ are obtained experimentally from Figure $4 \mathrm{~b}$ and $5 \mathrm{e}$.

$\therefore \mathrm{CT}$ dissociation rate $\frac{k_{d}}{k_{0}+k_{d}}=1-0.29=0.71$ gives $k_{0}=0.88 \mathrm{~ns}^{-1}$ and $k_{d}=2.11 \mathrm{~ns}^{-1}$

An additional energy factor due to applied reverse bias can be added in Arrhenius equation (Equation 3):

$$
k_{d}(V)=A \exp \left(\frac{-E_{a}-F \cdot d \cdot e}{k_{B} T}\right)=A \exp \left(\frac{-E_{a}-\frac{\Delta V}{\Delta l} \cdot e \cdot d}{k_{B} T}\right)
$$

where, the additional terms: $\Delta V$ is the applied electric potential across $\Delta l$, the thickness of the device, $d$ is the CT state size and $e$ is the elementary electronic charge. So, at $-5 \mathrm{~V}$ :

$$
\ln \frac{k_{d}}{k_{d 0}}=\ln \frac{2.11}{0.37}=\frac{-E_{a}-\frac{\Delta V}{\Delta l} \cdot e \cdot d}{k_{B} T}+\frac{E_{a}}{k_{B} T}=1.74
$$

$\therefore \boldsymbol{d}=\mathbf{0 . 9} \mathbf{n m}$, for $\Delta V=-5 \mathrm{~V}, \Delta l=100 \mathrm{~nm}$ and $T=298 \mathrm{~K}$. 


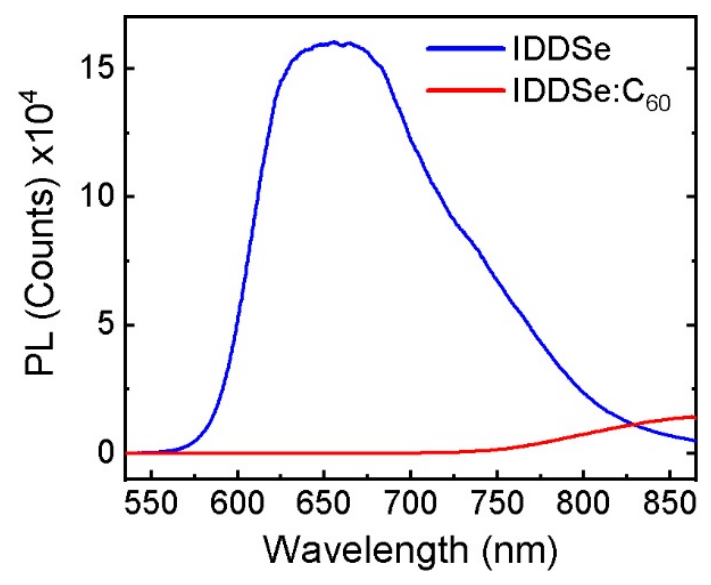

Figure S2. Absolute photoluminescence (PL) of neat IDDSe and blend IDDSe: $\mathrm{C}_{60}$ films. PL is obtained via $514 \mathrm{~nm}$ laser excitation.
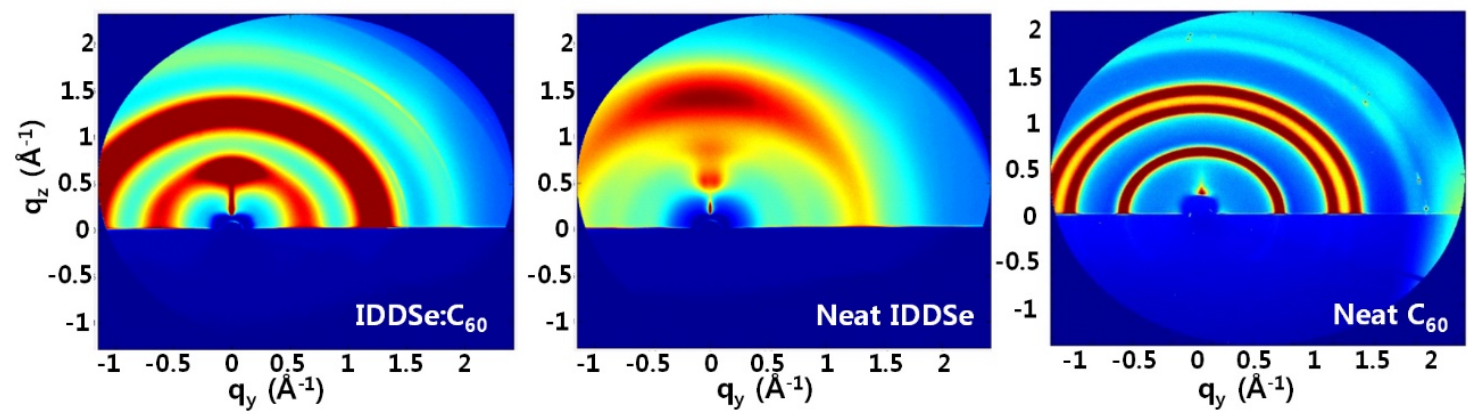

Figure S3. Grazing-incidence wide-angle x-ray scattering (GIWAXS) scans of thin films of the blend IDDSe: $\mathrm{C}_{60}$ compared to neat IDDSe and neat $\mathrm{C}_{60}$.
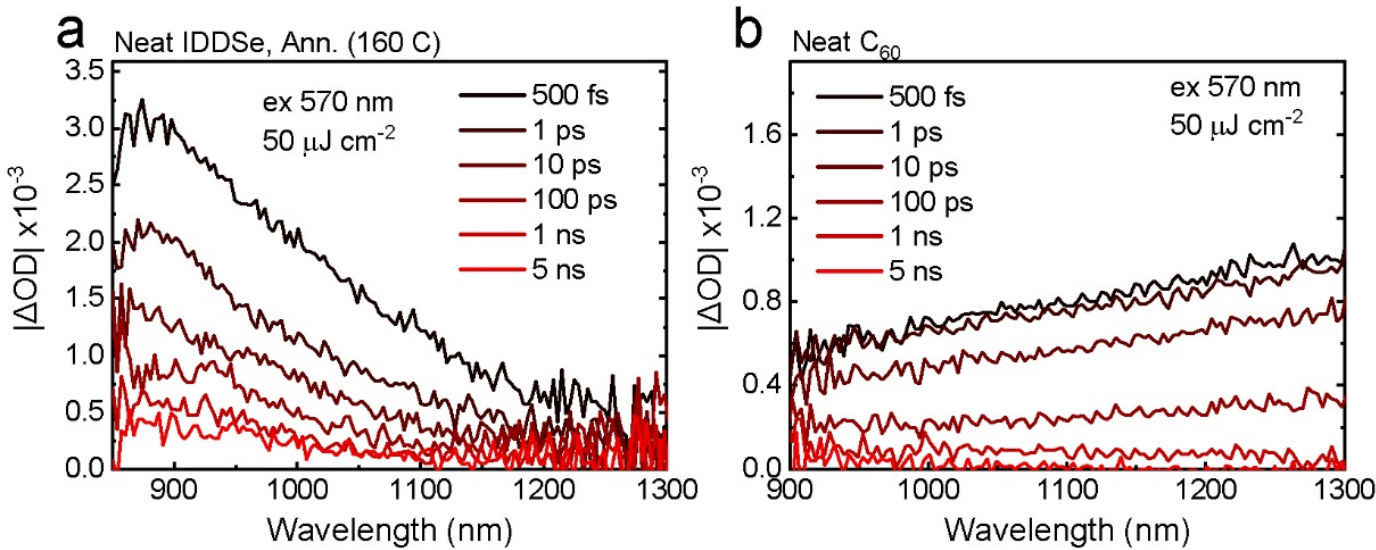

Figure S4. Fs-transient absorption spectra of (a) neat IDDSe annealed at $160{ }^{\circ} \mathrm{C}$ and (b) neat $\mathrm{C}_{60}$ film, excited at $570 \mathrm{~nm}$ with $50 \mu \mathrm{J} \mathrm{cm}^{-2}$. 

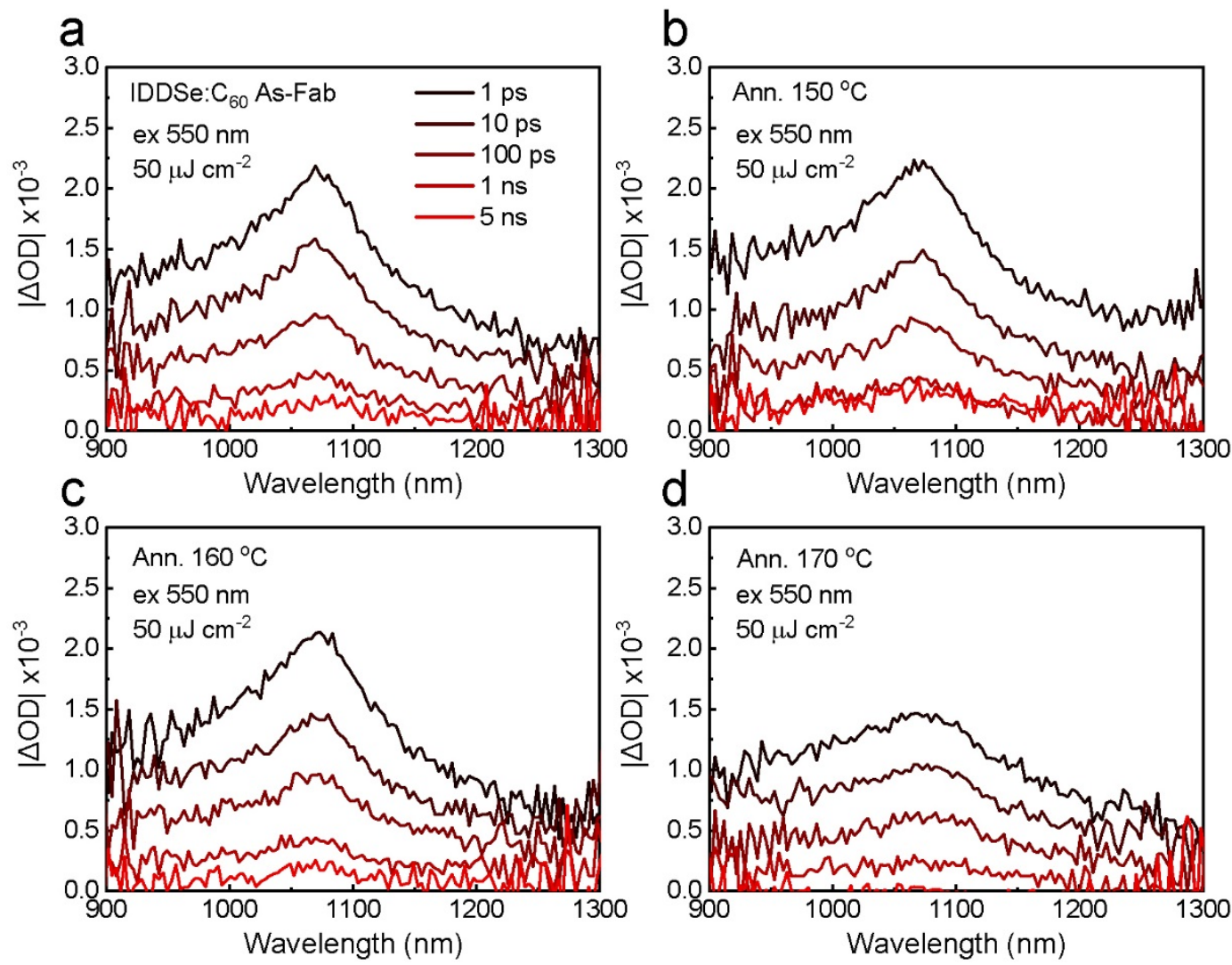

Figure S5. Fs-transient absorption spectra of IDDSe: $\mathrm{C}_{60}$ blend films after annealed at various temperatures, excited at $550 \mathrm{~nm}$ with $50 \mu \mathrm{J} \mathrm{cm}^{-2}$.
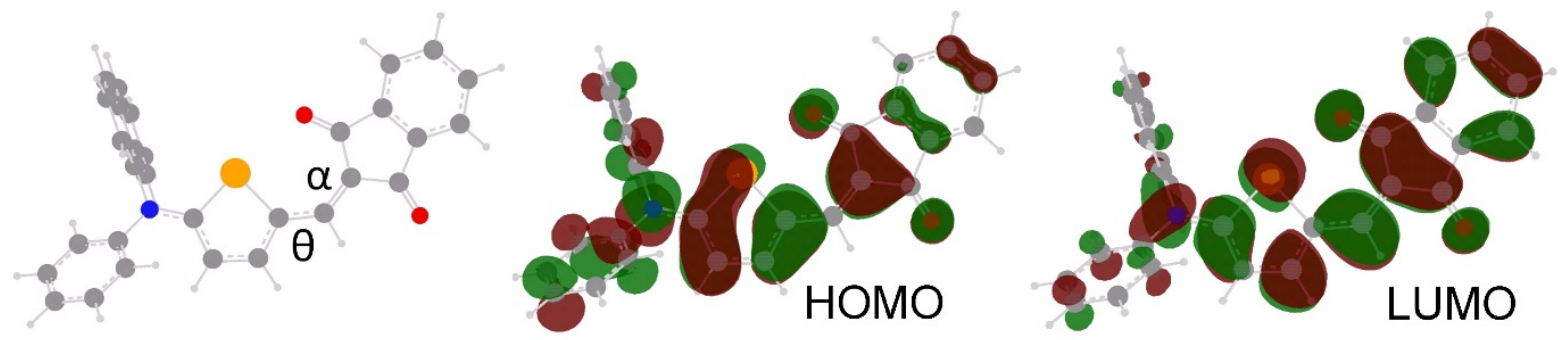

Figure S6. DFT optimized lowest potential energy structure of IDDSe molecule showing planar main conjugated backbone (with dihedral angles $\theta=0.4^{\circ}$ and $\alpha=0.9^{\circ}$ ). Also shown is HOMO and LUMO visualizations of the structure. Refer to DFT simulations in Experimental Section for details on the method of DFT calculation. 
a
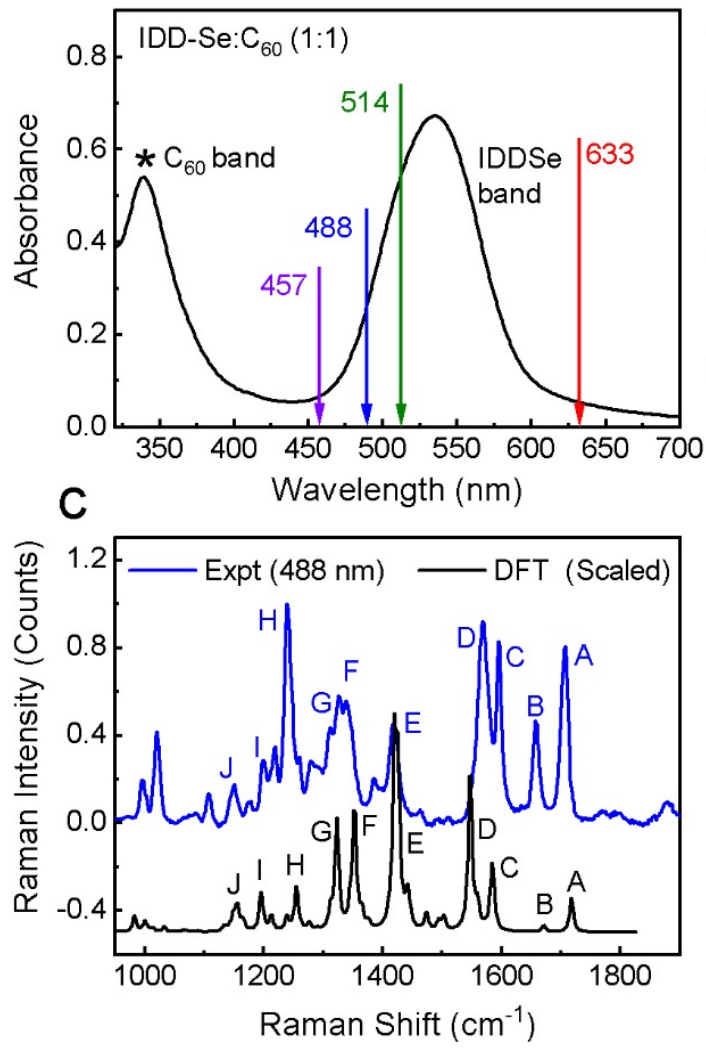

b

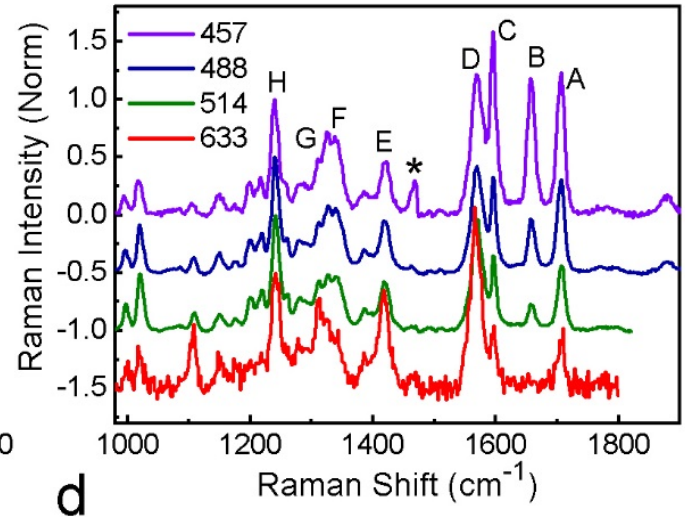

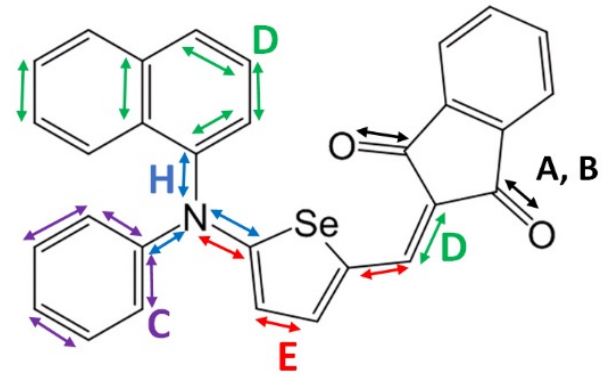

Figure S7. (a) Absorption spectrum of IDDSe: $\mathrm{C}_{60}$ film indicating specific laser excitations resonant $(457,488,514 \mathrm{~nm})$ and non-resonant $(633 \mathrm{~nm})$ to IDDSe absorption band (450-600 $\mathrm{nm})$. (b) Resonant and non-resonant Raman spectra of IDDSe: $\mathrm{C}_{60}$ film obtained via aforementioned laser excitations. Peaks A-H belong to IDDSe molecule and asterisked peak belongs to $\mathrm{C}_{6}$ molecule. (c) Comparison of experimental $(488 \mathrm{~nm}$ ) and DFT simulated Raman spectrum and assignments of IDDSe specific peaks. (d) Assignments of Raman peaks to their corresponding vibrational modes of IDDSe molecule.

Assignments of Raman peaks (A-H) with corresponding vibrational modes in IDDSe molecule, based on Supporting Figure S15 is shown in the table below:

\begin{tabular}{|l|l|}
\hline Peaks $\left(\mathbf{\pm 1} \mathbf{~ c m}^{-1}\right)$ & Vibrational Assignments \\
\hline H (1239) & C-N stretch \\
\hline Core Group & \multicolumn{2}{|l|}{} \\
\hline G (1326) & Whole $\pi$-conjugated backbone including fused benzene ring \\
\hline F (1339) & H-wagging of Selenophene and Alkene \\
\hline E (1417) & $\begin{array}{l}\text { C-C intraunit of Selenophene; extending to neighbouring C-N and C-C } \\
\text { interunit }\end{array}$ \\
\hline End Group & \multicolumn{2}{|l|}{} \\
\hline D (1569) & Naphthalene intraunit stretch (also includes strong Alkene stretch) \\
\hline C (1595) & Phenyl quadrant stretching mode, attached to Nitrogen \\
\hline B (1656) & C=O, anti-symmetric stretch \\
\hline A (1707) & C=O, symmetric stretch \\
\hline
\end{tabular}




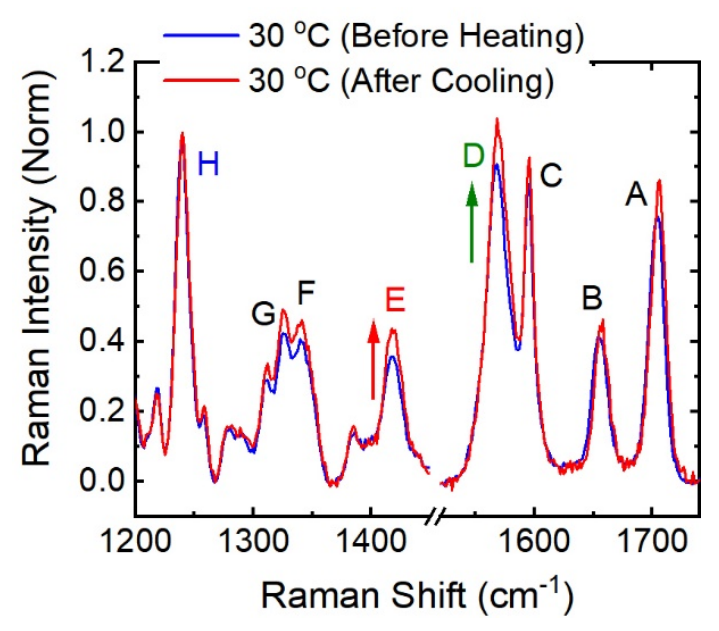

Figure S8. Normalized Raman of neat IDDSe film, taken at $30{ }^{\circ} \mathrm{C}$ (before heating and after cooling) showing the non-reversible effects of thermal annealing.
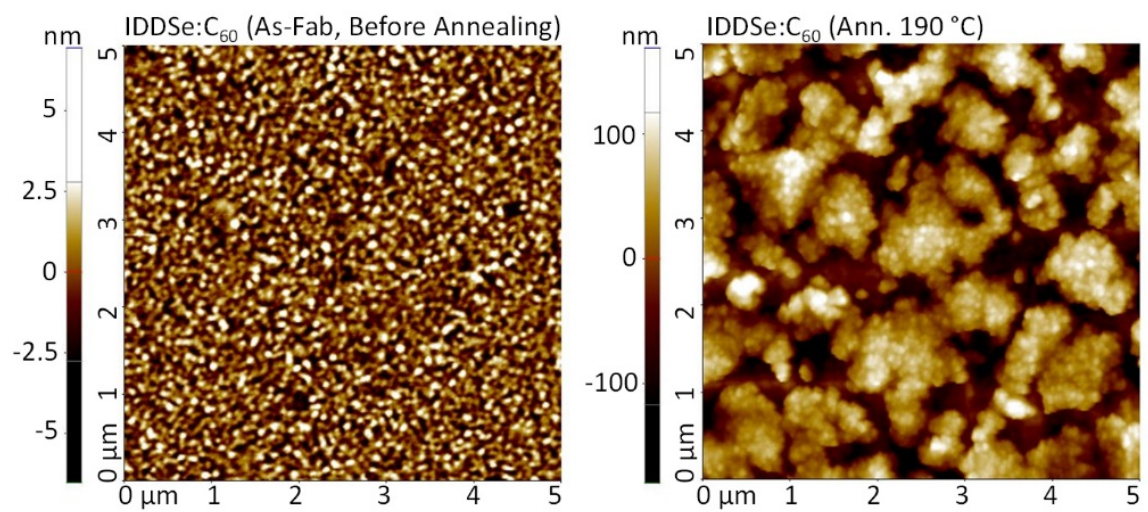

Figure S9. Atomic force microscopy scans of the IDDSe: $\mathrm{C}_{60}$ thin film before and after thermal annealing. The as-fabricated (before thermal annealing) and post-annealed (upon $190{ }^{\circ} \mathrm{C}$ annealing) blend show $\mathrm{rms}$ roughness of $0.6 \mathrm{~nm}$ and $59.6 \mathrm{~nm}$ respectively. The post-annealed blend film clearly shows micro-scale phase-segregation.
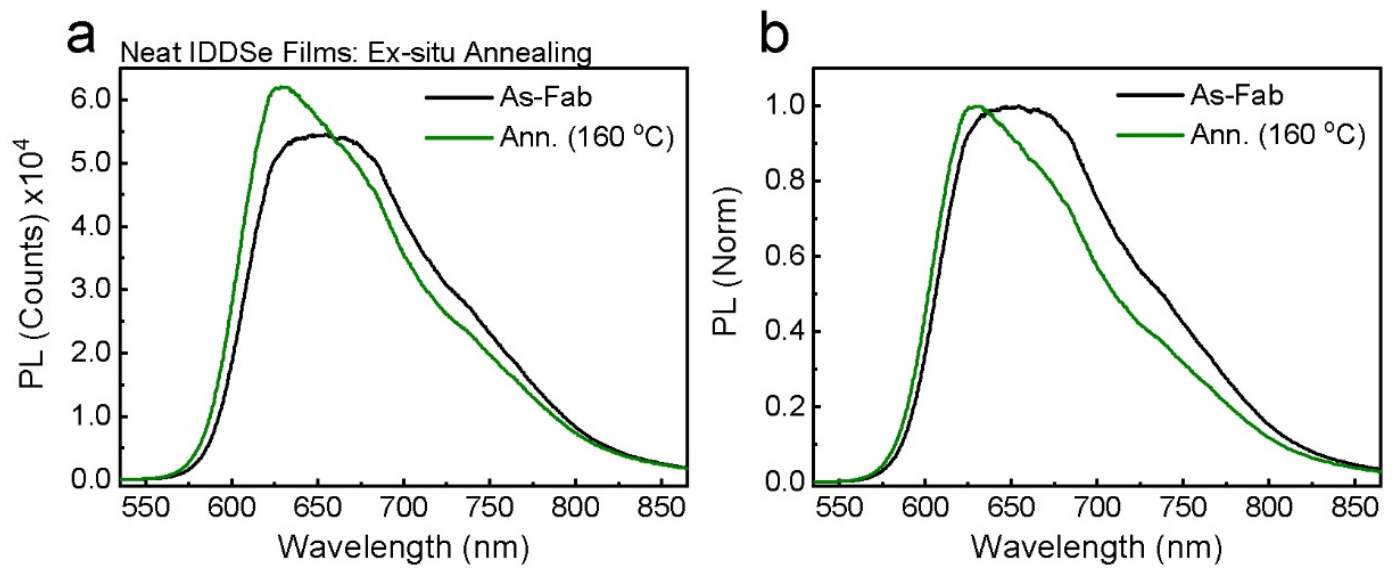

Figure S10. (a) Absolute and (b) Normalized photoluminescence (PL) of as-fabricated and annealed $\left(160^{\circ} \mathrm{C}\right)$ IDDSe films. PL is obtained via $514 \mathrm{~nm}$ laser excitation. 


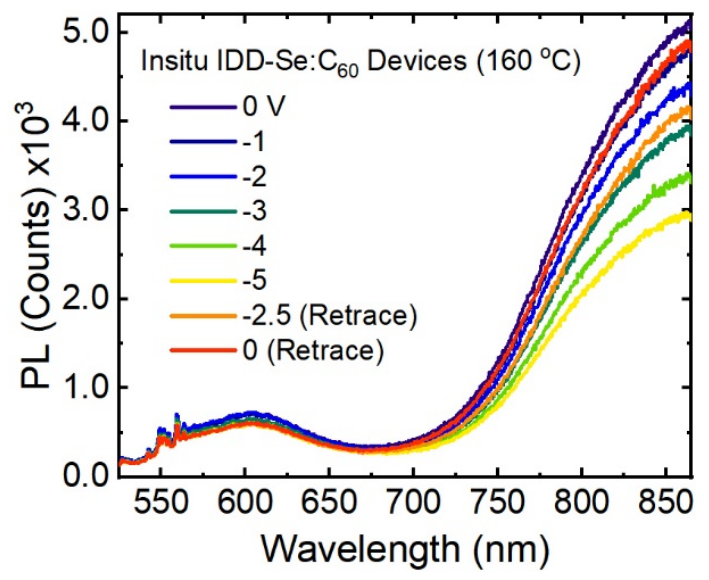

Figure S11. Reverse bias dependent photoluminescence (PL) of annealed $\left(160^{\circ} \mathrm{C}\right)$ IDDSe: $\mathrm{C}_{60}$ device. Note the recovery of PL upon retracing back to $0 \mathrm{~V}$. PL is obtained via $514 \mathrm{~nm}$ laser excitation.
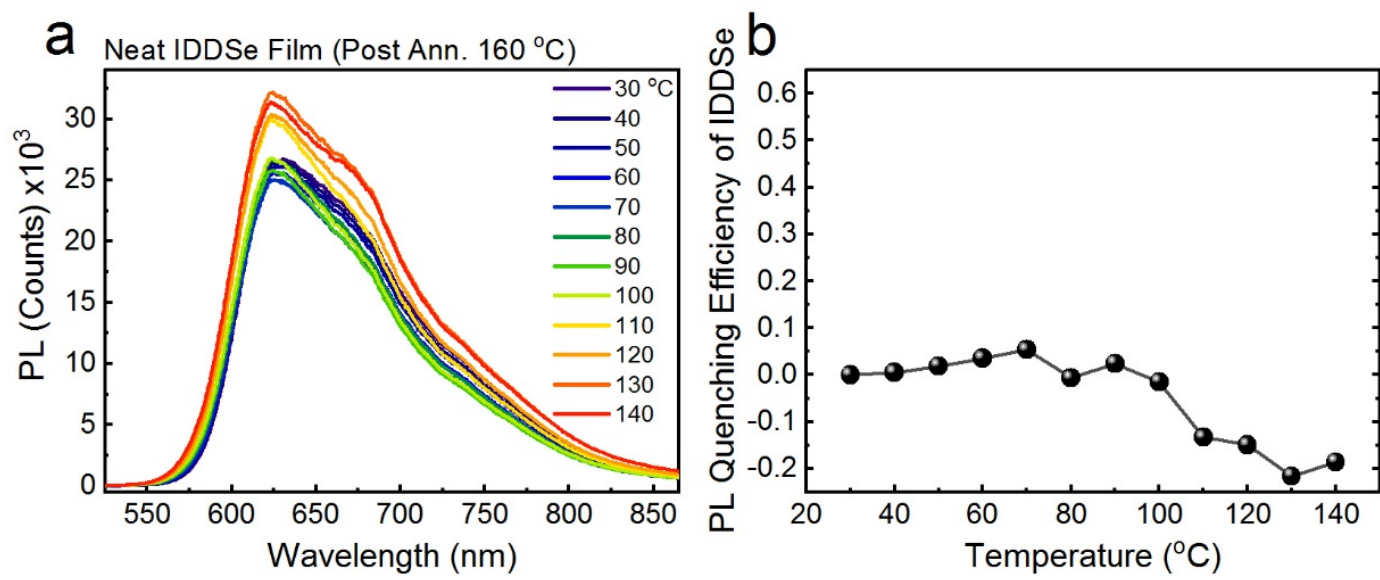

Figure S12. (a) Temperature dependent photoluminescence (PL) of annealed $\left(160^{\circ} \mathrm{C}\right)$ neat IDDSe film. (b) PL quenching efficiency of IDDSe emission as a function of temperature (with respect to emission at $30^{\circ} \mathrm{C}$ ) extracted at $625 \mathrm{~nm}$ emission. PL is obtained via $514 \mathrm{~nm}$ laser excitation.
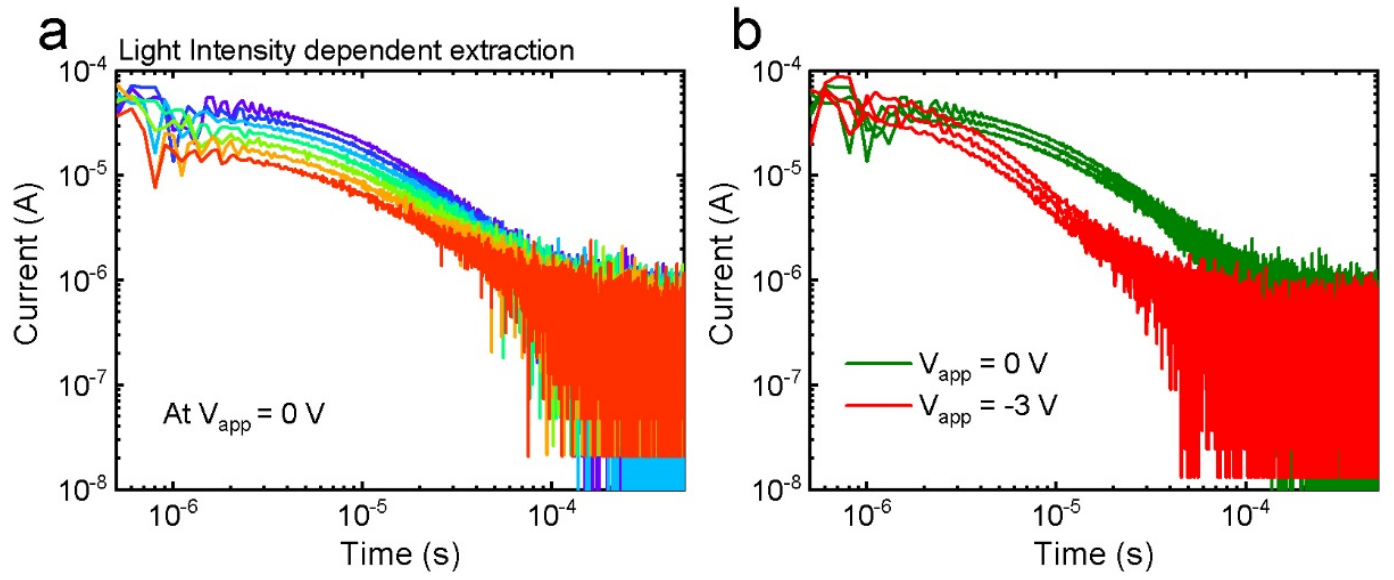

Figure S13. (a) Light intensity dependent photocurrent extraction transients for as-fabricated device extracted at short circuit. (b) Extraction transient at short circuit (green) and -3 V (red) respectively. 